\title{
Recommandations pour les analyses de cheveux dans les expertises médico-légales ${ }^{1}$
}

\section{Recommendations for hair testing in forensic cases ${ }^{1}$}

Society of Hair Testing (SoHT)

${ }^{1}$ Ces recommandations ont été publiées en anglais : Society of Hair Testing, Forensic Science International, Recommandations for hair testing in forensic cases, vol 145, pages 83-84, année 2004 (C), et sont proposées ici en français avec l'autorisation de Elsevier

\section{Introduction}

Le 7 octobre 2003, des représentants de 15 pays se sont réunis à Héraklion (Crète) pour discuter des problèmes rencontrés lors de l'analyse des stupéfiants dans les cheveux. Tous les représentants étaient, soit impliqués dans des analyses d'échantillons de cheveux dans leur propre laboratoire, ou les faisaient faire dans un autre laboratoire.

Les pays représentés étaient, par ordre alphabétique : l'Allemagne, le Canada, le Chili, l'Espagne, les Étatsunis, la France, la Grèce, l'Italie, le Luxembourg, la Norvège, la Pologne, le Portugal, le Royaume-Uni, la Suède et la Suisse.

Ce consensus n'a pas été finalisé à ce moment là. Le 24 janvier 2004, à Séville (Espagne), le comité de la SoHT s'est rassemblé et s'est mis d'accord sur les termes du consensus. Ils ont inclus les sections sur lesquelles l'assemblée s'était mise d'accord.

\section{Domaines de discussion}

\section{Prélèvement, envoi, conditionnement}

Ces thèmes ont été abordés dans un consensus précédent de la SoHT [1]

- L'échantillon doit être prélevé dans la région du vertex postérieur de la tête, le plus près possible du cuir chevelu, car c'est la région où la vitesse de croissance varie le moins. Si l'échantillon n'est pas prélevé à cet endroit, décrire l'emplacement du prélèvement. Les cheveux poussent, en général, à une vitesse approximative de $1 \mathrm{~cm}$ par mois.

- Le prélèvement n'a pas besoin d'être fait par un médecin.

- Noter la couleur, la longueur, l'endroit du prélèvement et tout traitement cosmétique que le cheveu aurait subi.

- Définir clairement la racine (partie proximale) de la pointe (partie distale) du cheveu.

- Si une analyse de cheveux par segmentation est nécessaire, attacher une mèche de cheveux avant de la couper.

- Le cheveu est le prélèvement idéal de phanères. Alternativement, les poils pubiens ou axillaires peuvent être prélevés si les cheveux ne sont pas disponibles.

- L'échantillon et tout fraction aliquote ou extrait doivent être manipulés avec précaution et conservés de façon à minimiser leur dégradation, la perte de stupéfiants ou leur contamination par une source extérieure. Les cheveux secs sont à conserver à l'abri de la lumière et à température ambiante.

- Une quantité suffisante d'échantillon doit être prélevée pour faire les premiers tests, ainsi que les analyses de confirmation ou de vérification si nécessaire. 
Le prélèvement d'une mèche de cheveux du diamètre d'un crayon de papier ou de plusieurs mèches du diamètre d'une paille est recommandé.

- Dans les cas post-mortem, les cheveux doivent être prélevés en début d'autopsie.

\section{Décontamination}

Quand une analyse de cheveux est faite pour identifier l'utilisation de stupéfiants, la limitation principale est la contamination externe, qui, si elle n'est pas éliminée, peut entraîner la confusion entre une exposition passive et une consommation réelle de stupéfiants. Le problème de la contamination externe doit être abordé par des méthodologies multiples et ne peut pas être résolu par la simple application d'une méthode unique.

- Les types de contamination possible doivent être considérés avant et pendant l'analyse. Ceux-ci peuvent inclure, entre autre, une exposition externe aux stupéfiants et une contamination au laboratoire.

- Une simple utilisation des valeurs limites est insuffisante car une contamination peut se produire à n'importe quel niveau.

- En général, une stratégie de décontamination doit inclure un solvant organique pour éliminer les substances grasses puis des lavages successifs à l'eau.

- Garder les solutions de lavages en cas d'analyses ultérieurs.

- Dans les cas d'autopsie ou d'exhumation, des traitements ultérieurs des cheveux au laboratoire sont parfois nécessaires, selon l'état de l'échantillon.

\section{Préparation des cheveux et extraction}

- Différentes procédures analytiques peuvent donner des résultats quantitatifs différents. Chaque laboratoire a le choix de désintégrer le cheveu avant l'extraction ou d'extraire le stupéfiant directement à partir du cheveu solide après une préparation adéquate.

- Des composés de dégradation peuvent être produits pendant le dosage. Pour estimer le degré de conversion/transformation, le laboratoire doit inclure des contrôles adéquats.

\section{Test de dépistage}

- Si un test de dépistage est utilisé, une méthode de validation appropriée incluant des calibrateurs et des contrôles dans la matrice cheveu doit être réalisée.

- Les stupéfiants retrouvés doivent être identifiés pour minimiser les faux négatifs.

\section{Critères pour l'analyse en spectrométrie de masse}

- La méthode doit être validée en suivant les bonnes pratiques de laboratoire.

- L'influence possible de standard interne de faible concentration doit être estimée et documentée.

- Pour des informations sur des critères valides pour l'analyse en spectrométrie de masse, se référer aux règles d'organismes scientifiques ou aux directives nationales.

\section{Classes spécifiques de stupéfiants}

Dans les cheveux, les stupéfiants et leurs métabolites doivent être analysés en utilisant des méthodes validées. Les limites de détection seront différentes selon les classes de stupéfiants. Quelques exemples pour des stupéfiants spécifiques sont présentés ci-dessous :

\section{Opiacés}

- Test immunochimique

- Une concentration de morphine ou de 6-acétylmorphine de $0,2 \mathrm{ng} / \mathrm{mg}$ doit donner un résultat positif.

- Analyse chromatographique

- Limite de quantification (LdQ) recommandée : $0,2 \mathrm{ng} / \mathrm{mg}$ pour chaque composé.

- La consommation d'héroïne doit être différenciée d'une utilisation de codéine ou de morphine par la présence de 6-acétylmorphine.

\section{Cocaïne}

- Test immunochimique

- Une concentration de cocaïne de $0,5 \mathrm{ng} / \mathrm{mg}$ doit donner un résultat positif.

- Analyse chromatographique

- LdQ recommandée : $<0,5 \mathrm{ng} / \mathrm{mg}$ pour la cocaïne ; $<0,05 \mathrm{ng} / \mathrm{mg}$ pour les autres composés.

- L'analyse chromatographique doit inclure la cocaïne et au moins un des composés suivants : la benzoylecgonine, le cocaéthylène, la norcocaïne ou l'ecgonine méthyl ester.

\section{Amphétamines}

- Test immunochimique

- Une concentration de $0,2 \mathrm{ng} / \mathrm{mg}$ doit produire séparément un résultat positif pour chacune des substances suivantes : la MDMA, la méthamphétamine, l'amphétamine, la MDEA ou la MDA.

- Analyse chromatographique

- LdQ recommandée : $<0,2 \mathrm{ng} / \mathrm{mg}$ pour chaque composé.

Remarque : les laboratoires doivent avoir connaissance du fait que la consommation de médicaments peut produire un résultat positif pour la méthamphétamine et l'amphétamine. 


\section{Cannabinoüdes}

- Test immunochimique

- Une concentration en THC de $0,1 \mathrm{ng} / \mathrm{mg}$ doit produire un résultat positif.

- Analyse chromatographique

- LOQ recommandée : THC $<0,1 \mathrm{ng} / \mathrm{mg}$ et THC$\mathrm{COOH}<0,2 \mathrm{pg} / \mathrm{mg}$.

- La confirmation de la présence de THC-COOH est obligatoire pour prouver une consommation de cannabinoïdes.

\section{Contrôle de qualité interne}

Le contrôle qualité interne pour les cheveux est plus difficile à mettre en place que pour les autres fluides biologiques homogènes car des échantillons contrôles surchargés ne peuvent pas remplacer les cheveux d'un toxicomane. Mais ils peuvent être substitués aux cheveux d'un toxicomane s'ils sont correctement préparés. - Une technique est de tremper pendant plusieurs jours des cheveux négatifs dans des solutions aqueuses contenant des stupéfiants à haute concentration, puis de complètement les laver avant de les sécher et de les analyser. Quand ils sont convenablement homogénéisés, ces échantillons surchargés peuvent être utilisés pour des études de précision, des contrôles qualités de routine, et comme contrôles internes de dégradation. Plusieurs types de cheveux devraient être utilisés.

- Bien que les contrôles puissent êtres des pools homogénéisés, on peut accepter leur substitution par des contrôles surchargés correctement préparés.

- Pour les stupéfiants endogènes, les contrôles peuvent être préparés en utilisant un milieu alternatif comme par exemple de la mélanine synthétique.

\section{Contrôle de qualité externe}

- Pour le contrôle qualité externe, le laboratoire doit s'inscrire à un programme de contrôle de qualité, où des échantillons de cheveux standards authentiques sont envoyés pour être testés.

- Le laboratoire doit analyser les échantillons du contrôle de qualité de la même manière que ses échantillons analysés en routine.

- Si un laboratoire n'exécute pas consciencieusement ces programmes externes, des sanctions doivent être prises.

- Dans le cas de la Society of Hair Testing, des laboratoires de référence seront nommés et leurs résultats seront utilisés comme méthode de référence et source de résultat.

- Les résultats de leur analyse sont compilés et comparés avec ceux des laboratoires de référence sélectionnés.

\section{Référence}

1. Anonyme, Society of Hair Testing, Forensic Sci. Int. 84 : 3-6 (1997). 